\title{
Beliefs and Intentions to Undergo Lung Cancer Screening among Korean Males
}

Nhung Cam Bui, MD, MPH'
Yoon Young Lee, MPH²
Mina Suh, MD, PhD²
Boyoung Park, MD, PhD',2
Hyunsoon Cho, PhD',
Yeol Kim, MD, PhD',
Kui Son Choi, PhD',

${ }^{1}$ Graduate School of Cancer Science and Policy, National Cancer Center, Goyang, ${ }^{2}$ National Cancer Control Institute, National Cancer Center, Goyang, Korea
Correspondence: Kui Son Choi, PhD Graduate School of Cancer Science and Policy, National Cancer Center, 323, Ilsan-ro, Ilsandong-gu, Goyang 10408, Korea

Tel: 82-31-920-2912

Fax: 82-31-920-2189

E-mail: kschoi@ncc.re.kr

Received August 21, 2017

Accepted November 15, 2017

Published Online November 16, 2017

\begin{abstract}
Purpose
Low-dose computed tomography (LDCT) has been reported as an effective screening method for lung cancer in high-risk populations. We aimed to examine willingness to be screened among Korean males using LDCT and to determine factors associated with lung cancer screening intentions (LCS) based on the Health Belief Model (HBM).
\end{abstract}

\section{Materials and Methods}

Data were obtained from the 2015 Korean National Cancer Screening Survey, a cross-sectional survey that utilized nationally representative random sampling. The survey included 1,730 male participants 40-74-year-old. Respondents were questioned regarding their willingness to undergo LCS and components of HBM. Factors associated with intentions to undergo screening were explored using logistic regression.

\section{Results}

Among participants, $65.2 \%$ were current smokers. Among high-risk subjects, $60.6 \%$ of men reported intentions to undergo LCS, compared to $49.9 \%$ of average-risk males. Men with higher perceived susceptibility in the average- and high-risk groups were, respectively, 1.63 (95\% confidence interval [Cl], 1.39 to 1.91) and 2.30 (95\% Cl, 1.14 to 4.63) times more likely to intend to undergo LCS compared to those with lower perceived barriers. Also, men in the average- and high-risk groups with higher perceived barriers to screening were, respectively, $0.79(95 \% \mathrm{Cl}, 0.68$ to 0.91$)$ and $0.52(95 \% \mathrm{Cl}, 0.29$ to 0.92$)$ times less likely to intend to undergo LCS compared to those with lower perceived barriers.

\section{Conclusion}

Tailored interventions designed to promote accurate perceptions of susceptibility and risk, as well as to reduce perceived barriers to screening, may effectively increase adherence to recommendations for LCS among high-risk Korean men.

\section{Introduction}

According to GLOBOCAN 2012, South Korea has the third highest lung cancer incidence worldwide. Among Korean men, lung cancer ranks the third most common cancer [1]. In 2011, the National Lung Screening Trial (NLST) reported that lung cancer screening by low-dose computed tomography (LDCT) significantly decreased lung cancer mortality (6.7\%) and overall mortality (20\%) among high-risk individuals [2].
Key words

Lung neoplasms, Early detection of cancer, Intention
According to Korean guidelines for lung cancer screening, annual LDCT screening is recommended to current smokers and ex-smokers (if less than 15 years have elapsed since smoking cessation) aged 55 to 74 years with a smoking history of 30 pack-years or more [3].

Despite of the effectiveness of lung cancer screening by LDCT, the harms thereof warrant consideration. In the NLST, $96.4 \%$ of the positive results in the LDCT group were false positive results across three rounds, and other studies reported that more than $90 \%$ of nodules were benign $[2,4,5]$. 
False-positive results lead to unnecessary examinations (both noninvasive and invasive tests) and psychological distress for participants. Additionally, cumulative radiation exposure from annual LDCT screening and additional test procedures could pose additional risk for lung cancer [6-8].

Few studies [9] have determined the feasibility of lung cancer screening in Korea. Accordingly, we conducted this cross-sectional study of healthy Korean males aged 40 years and older to examine intentions to be screened among the study population after exposure to information on the benefits and harms of lung cancer screening by LDCT. We also sought to determine factors associated with strong intentions to undergo lung cancer screening based on the Health Belief Model [10].

\section{Materials and Methods}

\section{Sample and sampling}

Data were obtained from subjects included in the 2015 Korean National Cancer Screening Survey (KNCSS). Since 2004, the KNCSS has been administered as an annual crosssectional survey to investigate screening rates among Koreans for five common cancers (gastric, liver, colorectal, breast, and cervix) through nationally representative random sampling. Stratified multistage random sampling based on resi- dent registration population data was conducted according to geographic area, age, and sex. The specific methods of this survey have been described elsewhere [11]. Eligibility criteria of the KNCSS included cancer-free men aged 40 years and older and women aged 20 years and older. Data were collected through face-to-face interviews conducted by a professional research agency. A total of 4,000 subjects participated in the KNCSS. The response rate after making contact was $66.0 \%$. Of the respondents, 1,730 men aged $\geq 40$ years were finally included in this study; since smoking prevalence was too low in women.

\section{Measures}

Each participant was asked to complete a questionnaire designed to collect information on socio-demographic characteristics, health-related characteristics, smoking status, and beliefs and intentions to undergo lung cancer screening via LDCT. Smoking status was measured by asking "Have you smoked at least 100 cigarettes in your entire life?" and "Do you now smoke cigarettes every day, some days, or not at all?" to classify subjects into current smokers, former smokers, and never smokers. Additionally, we also asked "number of packs of cigarettes smoked per day" and "number of years smoked" to assess pack-years. Based on the definition published by the Centers for Disease Control and Prevention [12], smoking status was defined as follows: (1) current smoker: respondents who reported smoking at least 100 cigarettes in their life time and who, at the time of survey,

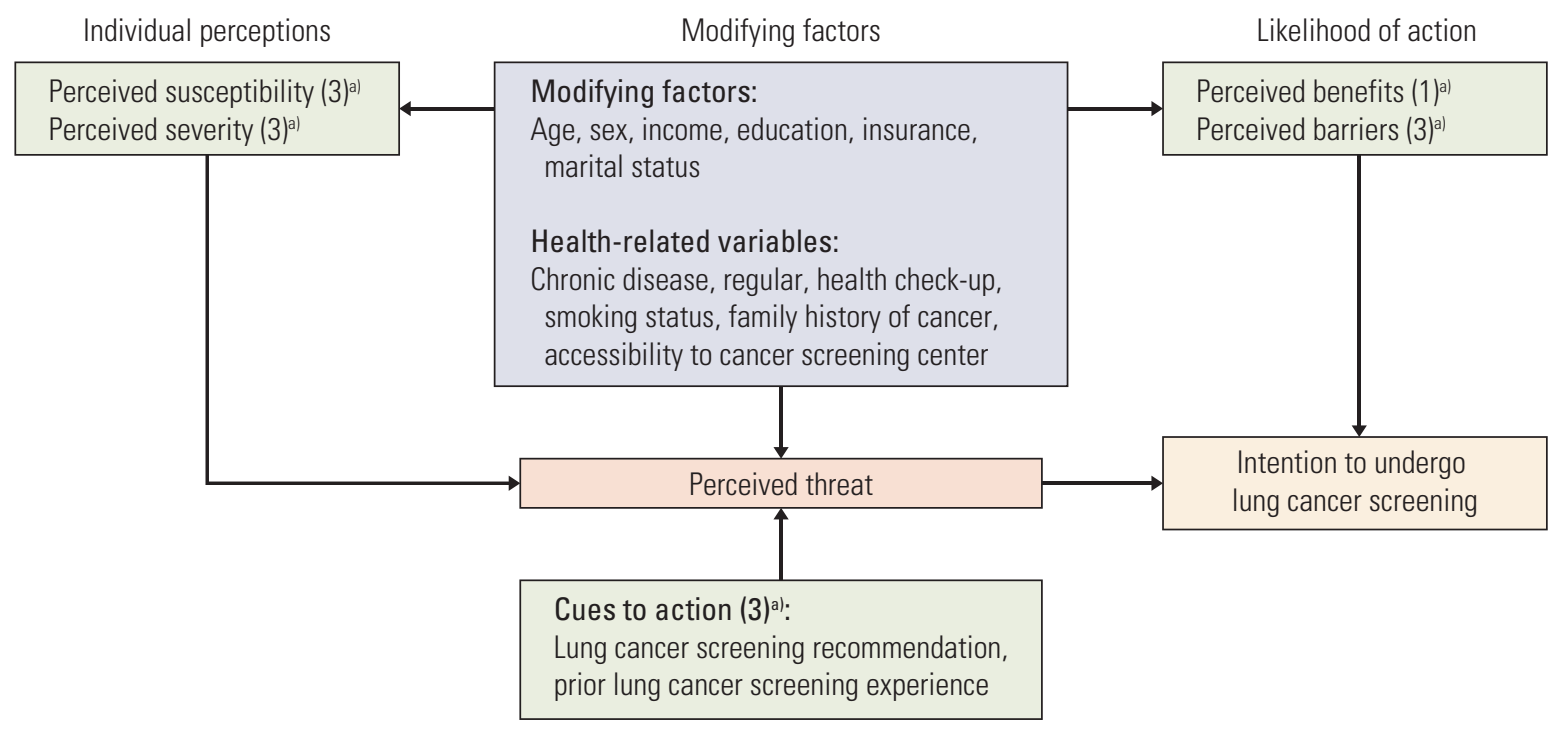

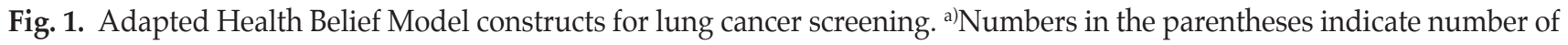
questions to measure each constructs. 
smoked either every day or some days; (2) former smoker: respondents who reported smoking at least 100 cigarettes in their life time and who, at the time of survey, did not smoke at all; and (3) never smoker: respondents who reported never having smoked 100 cigarettes.

Further, participants were divided in to high-risk and average-risk groups based on recommendations from Korean guidelines on lung cancer screening [3]. In the current study, the high-risk (eligible to be screened) group was defined as current smokers and ex-smokers (if less than 15 years had elapsed after smoking cessation) aged 55 to 74 years with a smoking-history of 30 pack-years or more. The average-risk group was defined as men aged 40-74 years not in the high-risk group.

The Health Belief Model (HBM) comprises five constructs: perceived susceptibility (three items), perceived severity (three items), perceived benefits (one item), perceived barriers (three items), and cues to action (three items). Each construct is measured using valid and reliable Likert scales with five response options ranging from " $1=$ not at all or never" to " $5=$ very much or always," except for cues to action. Adapted HBM constructs are described in Fig. 1.

To assess intentions to undergo lung cancer screening with LDCT, a photo of an individual receiving an LDCT examination was provided to each participant to describe the test. Participants were then explained the benefits of lung cancer screening as follows: "Lung cancer is the leading cause cancer death worldwide. For smokers who have smoked a pack of cigarettes per day over 30 years or who have smoked two packs of cigarettes per day over 15 years, LDCT examination may reduce lung cancer mortality by $20 \%$. However, smokers who have quit cigarettes for more than 15 years are not recommended to undergo screening." Then we asked "Do you want to undergo lung cancer screening through LDCT every year?" Also, we explained the harms of lung cancer screening, after which we asked the participants to describe their willingness to undergo annual lung cancer screening, as follows: "Whenever you undergo an LDCT scan for lung cancer screening, you might be exposed to radiation at a dose higher than recommended and you might also be misdiagnosed. Do you want to undergo lung cancer screening via LDCT every year?" There were four categories of responses: "Definitely yes," "Yes," "No," and "Never." Then, intentions to undergo lung cancer screening were categorized into two categories as follows: (1) strong intention, respondents who answered "Yes" or "Definitely yes" for both questions and (2) weak / no intention, respondents who answered "No" or "Never" for one of the two questions.

\section{Statistical analysis}

Descriptive statistics were used to analyze participant characteristics, smoking status, the five constructs of the $\mathrm{HBM}$, and intention to undergo lung cancer screening. Cronbach's alpha coefficient was calculated to examine the internal consistency of HBM constructs before conducting the analysis [13]. Principal axis factor analysis using a Varimax orthogonal rotation was conducted to classify each factor in the HBM model. A rotated factor loading of 0.40 was used to retain an item for further analysis. Factor scores were calculated using mean scores of retained questions in each factor (perceived susceptibility, perceived severity, perceived benefits, and perceived barriers). Due to two-point, Likerttype scales, cues to action were not calculated for a factor score, but categorized as dichotomous variables.

Univariate logistic regression models were applied to examine associations between intentions to undergo lung cancer screening with LDCT and variables in the conceptual framework. Then, variables with a p-value less than 0.10 from univariate analyses were selected for the multivariable analysis according to the average-risk and high-risk groups. Sub-group analyses for smoking status in the average-risk group were also conducted. All statistical analyses were performed using STATA software ver. 12 (Stata Corp. L.P., College Station, TX), and all p-values of $<0.05$ were considered statistically significant.

\section{Ethical statement}

Informed consent was obtained from all study participants, and this study was approved by the Institutional Review Board of the National Cancer Center, Korea.

\section{Results}

Table 1 shows the demographic characteristics of the study population. Of 1,730 participants, there were 160 male respondents eligible for lung cancer screening based on Korean lung cancer screening recommendations, accounting for $9.2 \%$ of the study population. High-risk participants were found to be less educated, of lower socioeconomic status, of worse health status, and more likely to live in a metropolitan area, comparing to those in the average-risk group.

Smoking-related characteristics are shown in Table 2. Overall, $65 \%$ of participants in the average-risk group were current smokers and $16.8 \%$ were former smokers. The mean smoking pack-years for smokers in the average-risk group was 13.6, while it is up to 43.1 among high-risk smokers. The high-risk group also showed greater total years of smoking (42.8 years) and fewer years since quitting smoking (9.6 years), compared to smokers in the average-risk group. 
Table 1. Demographic characteristics of the participants

\begin{tabular}{|c|c|c|c|c|}
\hline Characteristic & $\begin{array}{c}\text { Total } \\
(n=1,730)\end{array}$ & $\begin{array}{l}\text { Average } \\
(n=1,570)\end{array}$ & $\begin{array}{c}\text { High risk } \\
(\mathrm{n}=160)\end{array}$ & p-value \\
\hline \multicolumn{5}{|l|}{ Age group (yr) } \\
\hline $40-54$ & $1,016(58.7)$ & $1,016(64.7)$ & 0 & - \\
\hline $55-74$ & $714(41.3)$ & $554(35.3)$ & $160(100)$ & \\
\hline \multicolumn{5}{|l|}{ Marital status } \\
\hline Without spouse & $128(7.4)$ & $118(7.5)$ & $10(6.3)$ & 0.560 \\
\hline With spouse & $1,602(92.6)$ & $1,452(92.5)$ & $150(93.7)$ & \\
\hline \multicolumn{5}{|l|}{ Years of education } \\
\hline$<6$ & $61(3.5)$ & $47(2.3)$ & $14(8.8)$ & $<0.001$ \\
\hline $6-12$ & $1,005(58.1)$ & $888(56.6)$ & $117(73.1)$ & \\
\hline$\geq 13$ & $664(38.4)$ & $635(40.5)$ & $29(18.1)$ & \\
\hline \multicolumn{5}{|c|}{ Monthly household income } \\
\hline$\leq 2,999$ & $409(23.6)$ & $340(21.7)$ & $69(43.1)$ & $<0.001$ \\
\hline $3,000-4,999$ & $909(52.5)$ & $848(54.0)$ & $61(38.1)$ & \\
\hline$\geq 5,000$ & $412(23.8)$ & $382(24.3)$ & $30(18.8)$ & \\
\hline \multicolumn{5}{|c|}{ Private health insurance } \\
\hline Yes & 1,714 (99.1) & $1,556(99.1)$ & $158(1.3)$ & 0.652 \\
\hline No & $16(0.9)$ & $14(0.9)$ & $2(98.7)$ & \\
\hline \multicolumn{5}{|l|}{ Residency } \\
\hline Metropolitan & $776(44.9)$ & $659(42.0)$ & $117(73.1)$ & $<0.001$ \\
\hline Non-metropolitan & $954(55.1)$ & $911(58.0)$ & $43(26.9)$ & \\
\hline \multicolumn{5}{|c|}{ Self-reported health status } \\
\hline Good & $1,031(59.6)$ & $954(60.8)$ & $77(48.1)$ & 0.005 \\
\hline Normal & $640(37.0)$ & $566(36.0)$ & $74(46.3)$ & \\
\hline Poor & $59(3.4)$ & $50(3.2)$ & $9(5.6)$ & \\
\hline \multicolumn{5}{|c|}{ Regular health check-ups } \\
\hline Yes & $567(32.8)$ & $505(32.2)$ & $62(38.7)$ & 0.091 \\
\hline No & $1,163(67.2)$ & $1,065(67.8)$ & $98(61.3)$ & \\
\hline \multicolumn{5}{|c|}{ Family history of cancer } \\
\hline Yes & $264(15.3)$ & $237(15.1)$ & $27(16.9)$ & 0.551 \\
\hline No & $1,466(84.7)$ & $1,333(84.9)$ & $133(83.1)$ & \\
\hline \multicolumn{5}{|c|}{ Accessibility to cancer center } \\
\hline Convenient & $1,446(83.6)$ & $1,313(83.6)$ & $133(83.1)$ & 0.869 \\
\hline Inconvenient & $284(16.4)$ & $257(16.4)$ & $27(16.9)$ & \\
\hline
\end{tabular}

Values are presented as number $(\%) .{ }^{a}$ Comparing frequencies between average- and high-risk groups using chi-square test.

Table 2. Smoking-related characteristics of the participants according to the lung cancer risk

\begin{tabular}{|c|c|c|c|}
\hline Variable & $\begin{array}{c}\text { Total } \\
(n=1,730)\end{array}$ & $\begin{array}{c}\text { Average risk } \\
(\mathrm{n}=1,570)\end{array}$ & $\begin{array}{l}\text { High risk } \\
(n=160)\end{array}$ \\
\hline \multicolumn{4}{|l|}{ Smoking history } \\
\hline Never smoker & $312(18.0)$ & $312(19.9)$ & NA \\
\hline Former smoker & $290(16.8)$ & $239(15.2)$ & $51(31.9)$ \\
\hline Current smoker & $1,128(65.2)$ & $1,019(64.9)$ & $109(68.1)$ \\
\hline Smoking pack-years in former and current smokers & NA & $13.6 \pm 10.8$ & $43.1 \pm 17.4$ \\
\hline Years since quitting smoking among former smokers & NA & $11.9 \pm 7.4$ & $9.6 \pm 6.9$ \\
\hline Total smoking years in former and current smokers & NA & $31.9 \pm 8.4$ & $42.8 \pm 6.7$ \\
\hline Age at starting smoking & NA & $20.2 \pm 2.9$ & $20.1 \pm 2.3$ \\
\hline
\end{tabular}

Values are presented as number $(\%)$ or mean \pm standard deviation. NA, not applicable. 
Table 3. Descriptive statistics and Health Belief Model of lung cancer screening awareness

\begin{tabular}{|c|c|c|c|c|c|}
\hline \multirow{2}{*}{ Variable } & \multicolumn{2}{|c|}{ Average risk $(\mathrm{n}=1,570)$} & \multicolumn{2}{|c|}{ High-risk (n=160) } & \multirow{2}{*}{ p-value } \\
\hline & Agree $^{a}, \mathbf{n}(\%)$ & Mean \pm SD & Agree $\left.^{a}\right), n(\%)$ & Mean \pm SD & \\
\hline \multicolumn{6}{|l|}{ Perceived susceptibility } \\
\hline Chance of getting lung cancer in lifetime ${ }^{\mathrm{b})}$ & $388(24.7)$ & $2.92 \pm 0.86$ & $60(37.5)$ & $3.21 \pm 0.77$ & $<0.001$ \\
\hline $\begin{array}{l}\text { Possibility of getting lung cancer compared } \\
\text { to similar age group }{ }^{\text {b) }}\end{array}$ & $360(22.9)$ & $2.91 \pm 0.83$ & $56(35.0)$ & $3.23 \pm 0.72$ & $<0.001$ \\
\hline Often worry about getting lung cancerc) & $312(19.9)$ & $2.76 \pm 0.89$ & $40(25.0)$ & $2.96 \pm 0.92$ & 0.012 \\
\hline \multicolumn{6}{|l|}{ Perceived severity } \\
\hline Lung cancer leads to death' & $1,220(77.7)$ & $3.68 \pm 1.11$ & $135(84.4)$ & $3.78 \pm 0.94$ & 0.776 \\
\hline Lung cancer treatment is expensive ${ }^{c)}$ & $1,168(74.4)$ & $3.66 \pm 0.96$ & $120(75.0)$ & $3.64 \pm 0.85$ & 0.483 \\
\hline 5-Year survival is low ${ }^{c}$ & $1,037(66.1)$ & $3.46 \pm 1.09$ & $100(62.5)$ & $3.36 \pm 1.12$ & 0.325 \\
\hline \multicolumn{6}{|l|}{ Perceived benefits } \\
\hline LDCT helps detect and treat lung cancer ${ }^{\mathrm{b}}$ & $1,022(65.1)$ & $3.49 \pm 0.96$ & $108(67.5)$ & $3.51 \pm 0.82$ & 0.932 \\
\hline \multicolumn{6}{|l|}{ Perceived barriers } \\
\hline Concern about radiation exposure with $\mathrm{LDCT}^{\mathrm{b})}$ & $284(18.1)$ & $3.26 \pm 0.89$ & $25(15.6)$ & $3.29 \pm 0.92$ & 0.684 \\
\hline LDCT is painful ${ }^{\text {b) }}$ & 763 (48.6) & $2.76 \pm 1.07$ & $92(57.5)$ & $2.64 \pm 1.10$ & 0.187 \\
\hline \multicolumn{6}{|l|}{ Cues to action } \\
\hline Recommended to have lung cancer screening d) $^{\text {) }}$ & $121(7.7)$ & NA & $23(14.4)$ & NA & 0.004 \\
\hline Prior lung cancer screening experience ${ }^{\mathrm{d})}$ & $88(5.6)$ & NA & $17(10.6)$ & NA & 0.011 \\
\hline
\end{tabular}

SD, standard deviation; LDCT, low-dose computed tomography; NA, not applicable. ${ }^{a}$ Number of participants who answered "Agree" / "Somewhat" or "Totally agree" /"Very much", b)Comparing the mean scores between two groups using t test, "Testing differences in the distribution of variables between groups using Mann-Whitney test, ${ }^{\mathrm{d}}$ Two-point Likert-type scale; comparing agreement rates between groups using chi-square.

Table 3 demonstrates the mean scores of each question in the HBM regarding lung cancer screening stratified by risk group. From 13 questions, two items (one item to measure perceived barriers and the other to measure cues to action) were excluded due to factor analysis results. After orthogonal rotation, four factors were retained. Men in the high-risk group showed significantly higher perceived susceptibility scores than men in the average-risk group. Further, the highrisk group showed significantly higher percentages for agreement with cues to action items than the average-risk group.

Table 4 displays high intention rates among the averageand high-risk groups according to participant characteristics. Overall, $49.9 \%$ and $60.2 \%$ of the average- and high-risk participants, respectively, reported intentions to undergo lung cancer screening with LDCT. Among average-risk participants, former smokers showed the highest intention rate $(57.7 \%)$ to undergo screening, while it was lowest among non-smokers (39.1\%). In the average-risk group, men who were aged 40-54 years, living with spouse, of higher education level, living in a metropolitan area, and with convenient access to cancer screening units reported stronger intentions to undergo lung cancer screening. Regarding items in the HBM, men who had been recommended lung cancer screen- ing and had previously underwent lung cancer screening had stronger intentions to undergo screening than men who did not. Also, men with higher susceptibility and severity scores for lung cancer, as well as higher benefits scores for lung cancer screening, reported stronger intentions to undergo lung cancer screening, whereas men who had higher scores for barriers to lung cancer screening showed the weakest intentions. Except for variables concerning severity of lung cancer and benefits of lung cancer screening, these tendencies were similar in the high-risk group.

Factors associated with intentions to undergo lung cancer screening with LDCT according to risk groups are presented in Table 5. Among average-risk men, those who are living in a metropolitan area, had convenient access to a cancer screening unit, and current smokers were, respectively, more than 1.57 (95\% confidence interval [CI], 1.25 to 1.97$), 1.43$ (95\% CI, 1.07 to 1.91), and 1.35 (95\% CI, 1.02 to 1.79 ) times more likely to report intentions to undergo lung cancer screening compared to those of men living non-metropolitan areas, had inconvenient access to a cancer screening unit, and non-smokers. Additionally, those who had been recommended lung cancer screening (adjusted odds ratio [aOR], 2.60; 95\% CI, 1.54 to 4.38), had previously undergone lung cancer screening (aOR, 1.91; 95\% CI, 1.02 to 3.56), and had 
Table 4. Intentions to undergo lung cancer screening among male participants according to lung cancer risk

\begin{tabular}{|c|c|c|c|c|}
\hline \multirow{2}{*}{ Variable } & \multicolumn{2}{|c|}{ Average risk $(n=1,570)$} & \multicolumn{2}{|c|}{ High-risk (n=160) } \\
\hline & High intention ${ }^{\text {a) }}$ & p-value & High intention $^{\text {a) }}$ & p-value \\
\hline Total & $784(49.9)$ & & $97(60.6)$ & $0.10^{\mathrm{b})}$ \\
\hline \multicolumn{5}{|l|}{ Age group (yr) } \\
\hline $40-54$ & $539(53.1)$ & 0.001 & NA & NA \\
\hline $55-74$ & $245(44.2)$ & & $97(60.6)$ & \\
\hline \multicolumn{5}{|l|}{ Marital status } \\
\hline Without spouse & $46(39.0)$ & 0.013 & $7(70.0)$ & 0.741 \\
\hline With spouse & $738(50.8)$ & & $90(60.0)$ & \\
\hline \multicolumn{5}{|l|}{ Years of education } \\
\hline$<6$ & $19(40.4)$ & $<0.001$ & $8(57.1)$ & 0.953 \\
\hline $6-12$ & $397(44.7)$ & & $71(60.7)$ & \\
\hline$\geq 13$ & $368(58.0)$ & & $18(62.1)$ & \\
\hline \multicolumn{5}{|c|}{ Monthly household income } \\
\hline$\leq 2,999$ & $146(42.9)$ & 0.008 & $42(60.9)$ & 0.923 \\
\hline $3,000-4,999$ & $431(50.8)$ & & $36(59.0)$ & \\
\hline$\geq 5,000$ & $207(54.2)$ & & $19(63.3)$ & \\
\hline \multicolumn{5}{|c|}{ Private health insurance } \\
\hline Yes & $671(51.0)$ & 0.067 & $67(62.0)$ & 0.598 \\
\hline No & $113(44.7)$ & & $30(57.4)$ & \\
\hline \multicolumn{5}{|l|}{ Residency } \\
\hline Metropolitan & $376(57.1)$ & $<0.001$ & $79(67.5)$ & 0.003 \\
\hline Non-metropolitan & $408(44.8)$ & & $18(41.9)$ & \\
\hline \multicolumn{5}{|c|}{ Self-reported health status } \\
\hline Good & $483(50.6)$ & 0.612 & $43(55.8)$ & 0.406 \\
\hline Normal & $279(49.3)$ & & $49(66.2)$ & \\
\hline Poor & $22(44.0)$ & & $5(55.5)$ & \\
\hline \multicolumn{5}{|c|}{ Regular health check-ups } \\
\hline Yes & $246(48.7)$ & 0.504 & $45(72.6)$ & 0.014 \\
\hline No & $538(50.5)$ & & $52(53.1)$ & \\
\hline \multicolumn{5}{|c|}{ Family history of cancer } \\
\hline Yes & $132(55.7)$ & 0.054 & $15(55.6)$ & 0.554 \\
\hline No & $652(48.9)$ & & $82(61.7)$ & \\
\hline \multicolumn{5}{|c|}{ Access to cancer screening unit } \\
\hline Convenient & $677(51.6)$ & 0.004 & $82(61.7)$ & 0.554 \\
\hline Inconvenient & 107 (41.6) & & $15(55.6)$ & \\
\hline \multicolumn{5}{|l|}{ Smoking status } \\
\hline Never smoker & $122(39.1)$ & $<0.001$ & NA & \\
\hline Former smoker & $138(57.7)$ & & $33(64.7)$ & 0.470 \\
\hline Current smoker & $524(51.4)$ & & $64(58.7)$ & \\
\hline \multicolumn{5}{|c|}{ Ever recommended lung cancer screening } \\
\hline Yes & $96(79.3)$ & $<0.001$ & $20(87.0)$ & 0.005 \\
\hline No & $688(47.5)$ & & $77(56.2)$ & \\
\hline \multicolumn{5}{|c|}{ Prior lung cancer screening } \\
\hline Yes & $71(80.7)$ & $<0.001$ & $15(88.2)$ & 0.014 \\
\hline No & $713(48.1)$ & & $82(57.3)$ & \\
\hline Susceptibilityc) & $3.05 \pm 0.76$ & $<0.001$ & $3.25 \pm 0.66$ & 0.005 \\
\hline Severity ${ }^{\mathrm{d})}$ & $3.68 \pm 0.75$ & $<0.001$ & $3.62 \pm 0.68$ & 0.291 \\
\hline
\end{tabular}

(Continued to the next page) 
Table 4. Continued

\begin{tabular}{|c|c|c|c|c|}
\hline \multirow{2}{*}{ Variable } & \multicolumn{2}{|c|}{ Average risk $(\mathrm{n}=\mathbf{1}, \mathbf{5 7 0})$} & \multicolumn{2}{|c|}{ High-risk (n=160) } \\
\hline & High intention ${ }^{a}$ & p-value & High intention $^{\text {a) }}$ & p-value \\
\hline Benefits $^{c)}$ & $3.54 \pm 0.97$ & 0.062 & $3.60 \pm 0.82$ & 0.104 \\
\hline Barriers $^{\mathrm{c}}$ & $2.90 \pm 0.77$ & $<0.001$ & $2.87 \pm 0.80$ & $<0.001$ \\
\hline
\end{tabular}

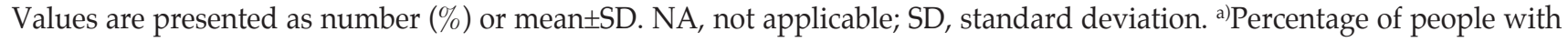
strong intentions among the average and high risk groups, ${ }^{\mathrm{b})} \mathrm{p}$-value when comparing strong intention rate between the average- and high-risk groups, ${ }^{\mathrm{c}}$ Comparing means between the strong and weak intention groups using $\mathrm{t}$ test, ${ }^{\mathrm{d}}$ Testing differences in the distribution of variables between the strong and weak intention groups using Mann-Whitney test.

Table 5. Factors associated with intention to undergo lung cancer screening among eligible and ineligible male screenees

\begin{tabular}{|c|c|c|c|c|}
\hline \multirow{2}{*}{ Variable } & \multicolumn{2}{|c|}{ Average risk } & \multicolumn{2}{|c|}{ High-risk } \\
\hline & aOR & $95 \% \mathrm{CI}$ & aOR & $95 \% \mathrm{CI}$ \\
\hline Age group (reference: $40-54 \mathrm{yr}$ ) & 0.83 & $0.65-1.06$ & NA & NA \\
\hline Marital status (reference: without spouse) & 1.25 & $0.82-1.90$ & 0.98 & $0.18-5.40$ \\
\hline \multicolumn{5}{|l|}{ Years of education (reference: $\leq 6$ ) } \\
\hline $6-12$ & 1.04 & $0.54-2.00$ & 0.98 & $0.24-4.12$ \\
\hline$\geq 13$ & 1.47 & $0.75-2.86$ & 1.28 & $0.21-7.64$ \\
\hline \multicolumn{5}{|l|}{ Monthly household income (reference: $\leq 2,999$ ) } \\
\hline $3,000-4,999$ & 1.07 & $0.81-1.43$ & 0.73 & $0.29-1.85$ \\
\hline$\geq 5,000$ & 1.20 & $0.86-1.66$ & 0.66 & $0.17-2.53$ \\
\hline Residency (reference: non-metropolitan) & 1.57 & $1.25-1.97$ & 5.98 & $2.34-15.23$ \\
\hline Family history of cancer (reference: no) & 1.10 & $0.82-1.49$ & 0.48 & $0.16-1.43$ \\
\hline Regular health checkups (reference: no) & 1.03 & $0.81-1.30$ & 2.88 & $1.21-6.82$ \\
\hline Accessibility to cancer center (reference: inconvenient) & 1.43 & $1.07-1.91$ & 0.96 & $0.33-2.73$ \\
\hline \multicolumn{5}{|l|}{ Smoking status (reference: never smoker) } \\
\hline Former smoker & 1.43 & $0.99-2.06$ & 1.00 & Reference \\
\hline Current smoker & 1.35 & $1.02-1.79$ & 0.67 & $0.28-1.57$ \\
\hline Ever recommended lung cancer screening (reference: no) & 2.60 & $1.54-4.38$ & 1.08 & $0.17-6.95$ \\
\hline Cues to action 2: prior lung cancer screening (reference: no) & 1.91 & $1.02-3.56$ & 10.20 & $0.92-112.8$ \\
\hline Susceptibility & 1.63 & $1.39-1.91$ & 2.30 & $1.14-4.63$ \\
\hline Severity & 1.13 & $0.97-1.31$ & 0.91 & $0.47-1.77$ \\
\hline Benefits & 1.02 & $0.91-1.14$ & 1.38 & $0.86-2.24$ \\
\hline Barriers & 0.79 & $0.68-0.91$ & 0.52 & $0.29-0.92$ \\
\hline
\end{tabular}

aOR, adjusted odd ratio; CI, confidence interval; NA, not available.

higher scores for perceived susceptibility (aOR, 1.63; 95\% CI, 1.39 to 1.91 ) were more likely to have intentions to undergo lung cancer screening compared to those who had not been recommended lung cancer screening, no previous lung cancer screening experience and lower scores for perceived susceptibility. In the high risk group, those who lived in a metropolitan area (aOR, 5.98; 95\% CI, 2.34 to 15.23), underwent regular health check-ups (aOR, 2.88; 95\% CI, 1.21 to 6.82), and higher perceived susceptibility (aOR, 2.30; 95\% CI,
1.14 to 4.63) were more likely to report intentions to undergo lung cancer screening compared to those who lived in nonmetropolitan area, irregular health check-ups and had lower perceived susceptibility. However, both average- and highrisk men with higher scores for barriers to lung cancer screening were, respectively, 0.79 (95\% CI, 0.68 to 0.91$)$ and 0.52 (95\% CI, 0.29 to 0.92 ) times less likely to have intentions to undergo lung cancer screening compared to those had lower perceived barriers scores. 


\section{Discussion}

This study was conducted using data from the KNCSS 2015, an annual cross-sectional study employing a nationally representative random sampling method. The average smoking pack-years of eligible screenees for lung cancer screening in this study (mean, 43.1 pack years; $95 \%$ CI, 40.5 to 45.9 ) was lower than that for individuals who participated in the NLST (NLST: 55.5 pack years in CT arm [2]). Previous studies have reported that current smokers in the United States tend to be from minority racial groups, of lower socioeconomic status, and be less educated $[14,15]$. In this study, we also found that high-risk individuals had significantly lower education levels, as well as lower household income, lower health status, and more chronic diseases than average-risk individuals. These would pose significant barriers to participating in lung cancer screening. Thus, invitation strategies need to be carefully devised to achieve equitable participation in lung cancer screening.

In the average-risk group, current (51.4\%) and former smokers $(57.7 \%)$ reported significantly stronger screening intentions than non-smokers $(39.1 \%)$. The intention rates in the current study were lower than those in previous studies conducted in the United States (59.3\%-98.2\%) [14-18] and other countries $[19,20]$. These differences can be partly explained by the definition of screening intention. In the current study, we conservatively categorized a person with intentions to undergo lung cancer as those who answered "Yes" or "Definitely yes" to two questions concerning their intentions for lung cancer screening. Up to now, no evidence has been reported on the efficacy of LDCT for lung cancer screening among average risk individuals. Nevertheless, in the present study, $50 \%$ of average-risk individuals indicated strong intentions to undergo lung cancer screening. Accordingly, we should stress the importance of educating the general population on the possible benefits, limitations, and known and uncertain harms, as well as screening criteria, for lung cancer. Further, for those current smokers who did not meet screening criteria, smoking cessation should be emphasized.

The rate of intentions to undergo lung cancer screening with LDCT in the high-risk group was higher than that in the average-risk group (60.6\% vs. $49.9 \%$ ). Albeit men in the highrisk group had the strongest intentions, this rate was still lower than those in other studies assessing willingness to be screened in high-risk populations $[16,19,21]$. The possible explanation is that, in the current study, we described both the benefits and the harms of LDCT to the participants, which might have weakened intentions. Among high-risk men, those who underwent regular health check-ups were more likely to have strong intentions to undergo screening for lung cancer than those who did not (aOR, 2.88; 95\% CI, 1.21 to 6.82 ). Also, men who had higher perceived susceptibility of getting lung cancer were more likely to have strong intentions to undergo lung cancer screening (aOR, 2.30; 95\% CI, 1.14 to 4.63 ) than those with lower perceived susceptibility. However, men facing greater perceived barriers to screening reported weaker intentions to undergo lung cancer screening (aOR, 0.52; 95\% CI, 0.29 to 0.92 ) than those with lower perceived barriers. These findings are similar to previous studies on high-risk populations $[16,19,21,22]$. In particular, our finding supports a previous study in which fear of radiation exposure was found to be related to weaker intentions to be screened among smokers [18].

Although perceived susceptibility was found to be a significant factor boosting intentions for lung cancer screening, only around $35 \%$ of men in the high-risk group believed that they had a higher risk of getting lung cancer than people of similar age. Also, $25 \%$ of men in the high-risk group said that they worried about lung cancer. In other words, around 65\%$75 \%$ of men in the high-risk group did not perceive that they were highly susceptible to lung cancer. These low perceptions of lung cancer risk may be a factor affecting hesitation with lung cancer screening in high-risk individuals. Thus, there may be a need to educate people on determining their lung cancer risk in order to increase adherence with lung cancer prevention and early detection programs.

Perceived barriers were found to be a significant factor affecting weaker intentions to undergo lung cancer screening. In the current study, approximately $58 \%$ of men in the high-risk group perceived that the LDCT test would be painful, compared to $49 \%$ of men in the average-risk group. Even though we explained the features of LDCT to participants using a picture card, on average, half of the study participants worried about pain during the examination. On the contrary, there was less concern for radiation exposure from LDCT in both groups. This suggests that education about the LDCT test is required. We also considered the cost of LDCT as a barrier to lung cancer screening; however, it was excluded from the final analysis due to low factor loading in factor analysis. Nevertheless, if lung cancer screening can be covered by national cancer screening programs, screening costs would not be a potential barrier to lung cancer screening.

Among other modifiable factors, residency area was associated with lung cancer screening intentions in both averagerisk and high-risk groups. In particular, in high-risk men, those who lived in a metropolitan area showed higher odds of intending to undergo lung cancer screening (aOR, 5.98; 95\% CI, 2.34 to 15.23) than those lived in a non-metropolitan area. In the average-risk group, men with convenient access to a cancer screening center were more likely to have strong intentions to undergo lung cancer screening. This suggests 
that greater availability to LDCT may increase intentions to undergo the test, which could lead to a moral hazard if LDCT is widely provided without considering exposure of lung cancer risk [23]. Thus, comprehensive education on the benefits and harms of LDCT and eligibility criteria for lung cancer screening should be provided to the general population. Interestingly, in the average-risk group, cues to action in HBM were significantly associated with intentions to undergo lung cancer screening, whereas in the high-risk group, they were not. Particularly, in the average-risk group, those who had ever been recommended lung cancer screening were 2.60 times more likely to have intentions to undergo lung cancer screening than those who had never been recommended. This suggests that a doctor's recommendation has a tremendous influence on intentions to be screened in average-risk populations. Thus, it is necessary for doctors to provide clear information on lung cancer screening, especially for screening targets.

This study was conducted to examine intentions to undergo lung cancer screening among the general Korean male population. We used nationwide data in the context of a stratified, multistage, random sampling procedure and were representative of the general Korean male population. We estimated that there were 160 eligible screenees for lung cancer screening, accounting for $22.4 \%$ of people aged from 55-74 years in this study. Furthermore, we attempted to provide balanced information on the benefits and harms of LDCT, and tried to assess participants' decision making for lung cancer screening based on this information. Nevertheless, our study has several limitations. First, data on demographic characteristics and smoking status were selfreported; therefore, although the interviewers received standardized training, recall and interviewer biases remain likely. Second, we employed a cross-sectional design, such that it was not possible to discern causal relationships.
Finally, we examined intentions rather than actual behaviors. We could not calculate the percentage of the population who would actually undergo the screening test. Future studies could benefit from recording this information for use in analyses.

In the current study, among the five constructs of the HBM, perceived susceptibility was found to be a significant factor affecting lung cancer screening intentions. This suggests a need to educate people on their lung cancer risk. Also, perceived barriers (concerns for radiation exposure and pain with LDCT) appeared to negatively affect lung cancer screening intentions. Our findings provide directions for developing psychological interventions to promote the use of LDCT for lung cancer screening. Interventions designed to promote accurate perceived susceptibility and risk, as well as to reduce perceived barriers, may effectively increase adherence with lung cancer screening recommendations among high-risk individuals. Furthermore, educating lower-risk individuals on their relative risk of developing lung cancer, as well as the harms of LDCT for lung cancer screening, is needed. In particular, smoking cessation interventions for current smokers, both those who meet lung cancer screening criteria and those who do not, should be provided within the context of LDCT cancer screening.

\section{Conflicts of Interest}

Conflict of interest relevant to this article was not reported.

\section{Acknowledgments}

The authors would like to thank the staff of the National Health Insurance Service. This study was supported by a Grant-in-Aid for Cancer Research and Control from the National Cancer Center of Korea (\#1310232 and \#1610401).

\section{References}

1. Ferlay J, Soerjomataram I, Ervik M, Dikshit R, Eser S, Mathers C, et al. GLOBOCAN 2012 v1.0. Cancer incidence and mortality worldwide: IARC CancerBase No. 11. Lyon: International Agency for Research on Cancer; 2013.

2. National Lung Screening Trial Research Team, Aberle DR, Adams AM, Berg CD, Black WC, Clapp JD, et al. Reduced lung-cancer mortality with low-dose computed tomographic screening. N Engl J Med. 2011;365:395-409.

3. Jang SH, Sheen S, Kim HY, Yim HW, Park BY, Kim JW, et al. The Korean guideline for lung cancer screening. J Korean Med Assoc. 2015;58:291-301.
4. Bach PB, Mirkin JN, Oliver TK, Azzoli CG, Berry DA, Brawley $\mathrm{OW}$, et al. Benefits and harms of CT screening for lung cancer: a systematic review. JAMA. 2012;307:2418-29.

5. Gohagan JK, Marcus PM, Fagerstrom RM, Pinsky PF, Kramer BS, Prorok PC, et al. Final results of the Lung Screening Study, a randomized feasibility study of spiral CT versus chest $X$-ray screening for lung cancer. Lung Cancer. 2005;47:9-15.

6. Mettler FA Jr, Huda W, Yoshizumi TT, Mahesh M. Effective doses in radiology and diagnostic nuclear medicine: a catalog. Radiology. 2008;248:254-63.

7. National Lung Screening Trial Research Team, Aberle DR, 
Berg CD, Black WC, Church TR, Fagerstrom RM, et al. The National Lung Screening Trial: overview and study design. Radiology. 2011;258:243-53.

8. Smith-Bindman R, Lipson J, Marcus R, Kim KP, Mahesh M, Gould R, et al. Radiation dose associated with common computed tomography examinations and the associated lifetime attributable risk of cancer. Arch Intern Med. 2009;169:2078-86.

9. Nhung BC, Lee YY, Yoon H, Suh M, Park B, Jun JK, et al. Intentions to undergo lung cancer screening among Korean men. Asian Pac J Cancer Prev. 2015;16:6293-8.

10. Rosenstock IM. The health belief model and preventive health behavior. Health Educ Monogr. 1974;2:354-86.

11. Suh M, Choi KS, Park B, Lee YY, Jun JK, Lee DH, et al. Trends in cancer screening rates among Korean men and women: results of the Korean National Cancer Screening Survey, 20042013. Cancer Res Treat. 2016;48:1-10.

12. Centers for Disease Control and Prevention (CDC). State-specific secondhand smoke exposure and current cigarette smoking among adults: United States, 2008. MMWR Morb Mortal Wkly Rep. 2009;58:1232-5.

13. O'Rourke N, Hatcher L. A step-by-step approach to using SAS for factor analysis and structural equation modeling. 2nd ed. Cary, NC: SAS Institute Inc.; 2013.

14. Silvestri GA, Nietert PJ, Zoller J, Carter C, Bradford D. Attitudes towards screening for lung cancer among smokers and their non-smoking counterparts. Thorax. 2007;62:126-30.

15. Tanner NT, Egede LE, Shamblin C, Gebregziabher M, Silvestri
GA. Attitudes and beliefs toward lung cancer screening among US Veterans. Chest. 2013;144:1783-7.

16. Cataldo JK. High-risk older smokers' perceptions, attitudes, and beliefs about lung cancer screening. Cancer Med. 2016;5: 753-9.

17. Schnoll RA, Bradley P, Miller SM, Unger M, Babb J, Cornfeld M. Psychological issues related to the use of spiral CT for lung cancer early detection. Lung Cancer. 2003;39:315-25.

18. Jonnalagadda S, Bergamo C, Lin JJ, Lurslurchachai L, Diefenbach M, Smith C, et al. Beliefs and attitudes about lung cancer screening among smokers. Lung Cancer. 2012;77:526-31.

19. Flynn AE, Peters MJ, Morgan LC. Attitudes towards lung cancer screening in an Australian high-risk population. Lung Cancer Int. 2013;2013:789057.

20. Patel D, Akporobaro A, Chinyanganya N, Hackshaw A, Seale C, Spiro SG, et al. Attitudes to participation in a lung cancer screening trial: a qualitative study. Thorax. 2012;67:418-25.

21. Ali N, Lifford KJ, Carter B, McRonald F, Yadegarfar G, Baldwin DR, et al. Barriers to uptake among high-risk individuals declining participation in lung cancer screening: a mixed methods analysis of the UK Lung Cancer Screening (UKLS) trial. BMJ Open. 2015;5:e008254.

22. Young RP, Hopkins RJ. Increasing smokers' risk perception improves CT screening participation. Thorax. 2012;67:834-5.

23. Manning WG, Marquis MS. Health insurance: the tradeoff between risk pooling and moral hazard. J Health Econ. 1996;15:609-39. 